\title{
A PROPERTY OF INFINITELY DIFFERENTIABLE FUNCTIONS
}

\author{
HA HUY BANG
}

(Communicated by R. Daniel Mauldin)

\begin{abstract}
The existence of $\lim _{n \rightarrow \infty}\left\|f^{(n)}\right\|_{p}^{1 / n}$ for an arbitrary function $f(x) \in$ $C^{\infty}(\mathbf{R})$ such that $f^{(n)}(x) \in L^{p}(\mathbf{R}), n=0,1, \ldots \quad(1 \leq p \leq \infty)$ and the concrete calculation of $\lim _{n \rightarrow \infty}\left\|f^{(n)}\right\|_{p}^{1 / n}$ are shown.
\end{abstract}

Theorem 1. Let $1 \leq p \leq \infty$ and $f(x) \in C^{\infty}(\mathbf{R})$ such that $f^{(n)}(x) \in L^{p}(\mathbf{R})$, $n=0,1, \ldots$. Then there always exists the limit

$$
d_{f}=\lim _{n \rightarrow \infty}\left\|f^{(n)}\right\|_{p}^{1 / n}
$$

and moreover

$$
d_{f}=\sigma_{f}=\sup \{|\xi|: \xi \in \operatorname{supp} \tilde{f}(\xi)\},
$$

where the last equality is the definition of $\sigma_{f}$ and $\tilde{f}(\xi)$ is the Fourier transform of the function $f(x)$.

Proof. We shall begin by showing that there exists the limit

$$
d_{f}=\lim _{n \rightarrow \infty}\left\|f^{(n)}\right\|_{p}^{1 / n} .
$$

Without loss of generality we may assume that $\|f\|_{p}=1$. Then using the Kolmogoroff-Stein theorem [1, 2], we have

$$
\left\|f^{(k)}\right\|_{p}^{n} \leq(\pi / 2)^{n}\left\|f^{(n)}\right\|_{p}^{k}, \quad 0<k<n,
$$

for any $n=2,3, \ldots$, and hence

$$
\left\|f^{(k)}\right\|_{p}^{1 / k} \leq(\pi / 2)^{1 / k}\left\|f^{(n)}\right\|_{p}^{1 / n}, \quad 0<k<n .
$$

By (2) it follows that

$$
\left\|f^{(k)}\right\|_{p}^{1 / k} \leq(\pi / 2)^{1 / k} \underline{\lim }_{n \rightarrow \infty}\left\|f^{(n)}\right\|_{p}^{1 / n}
$$

Received by the editors March 10,1987. The results presented in this paper were obtained by the author under the supervision of Professor Tran Duc Van at The Institute of Mathematics, Hanoi.

1980 Mathematics Subject Classification (1985 Revision). Primary 26A93.

* The Fourier transform is in the sense of [3]. 
for any $k=1,2, \ldots ;$ therefore

$$
\varlimsup_{k \rightarrow \infty}\left\|f^{(k)}\right\|_{p}^{1 / k} \leq \varliminf_{n \rightarrow \infty}\left\|f^{(n)}\right\|_{p}^{1 / n}
$$

Equation (1) is immediate from (3).

Further, we shall prove that $d_{f}=\sigma_{f}$. We first observe that

$$
d_{f} \leq \sigma_{f}
$$

It is enough to show (4) for $\sigma_{f}<\infty$. Then using $f \in \mathscr{S}^{\prime}$ (this follows from $f \in L^{p}(\mathbf{R})$ ) and the well-known Paley-Wiener-Schwartz theorem, we obtain that $f$ is an analytic function of exponential type $\leq \sigma_{f}$. Hence by the BernsteinNikolsky inequality [3, p. 115] it follows that

$$
\left\|f^{(n)}\right\|_{p} \leq \sigma_{f}^{n}\|f\|_{p}, \quad n=0,1, \ldots,
$$

and (4) is an immediate consequence of the last inequalities.

Finally, we claim that $d_{f} \geq \sigma_{f}$. We divide the proof into two cases.

Case 1. $p=\infty$. Assume the contrary, that $d_{f}<\sigma_{f}$. Then there exist numbers $M<\infty, \sigma<\sigma_{f}$ such that

$$
\left\|f^{(n)}\right\|_{\infty} \leq M \sigma^{n}, \quad n=0,1, \ldots
$$

Therefore, using the inverse theorem of Bernstein we have that $f$ is an analytic function of exponential type $\leq \sigma<\infty$. Consequently, it follows from Schwartz's theorem [3, p. 110] that $\operatorname{supp} \widetilde{f}(\xi) \subset\{\xi:|\xi| \leq \sigma\}$. This contradicts the assumption that $\sigma<\sigma_{f}$.

Case 2. $1 \leq p<\infty$. Let

$$
f_{k}(x)=k \int_{0}^{1 / k} f(x+t) d t, \quad k=1,2, \ldots .
$$

Then by Jensen's inequality we obtain

$$
\left|f_{k}^{(n)}(x)\right|^{p} \leq k \int_{0}^{1 / k}\left|f^{(n)}(x+t)\right|^{p} d t, \quad k=1,2, \ldots,
$$

for any $n=0,1, \ldots$; therefore,

$$
\left\|f_{k}^{(n)}\right\|_{\infty} \leq k^{1 / p}\left\|f^{(n)}\right\|_{p}, \quad n=0,1, \ldots k=1,2, \ldots
$$

On the other hand, Case 1 shows that

$$
\sigma_{f_{k}}=\lim _{n \rightarrow \infty}\left\|f_{k}^{(n)}\right\|_{\infty}^{1 / n}, \quad k=1,2, \ldots
$$

Combining (6) and (7) yields

$$
\sigma_{f_{k}} \leq \lim _{n \rightarrow \infty}\left\|f^{(n)}\right\|_{p}^{1 / n}=d_{f}, \quad k=1,2, \ldots
$$

Consequently, to complete the proof it remains to show that

$$
\sigma_{f} \leq \varliminf_{k \rightarrow \infty}^{\lim } \sigma_{f_{k}}
$$


and therefore the problem is now reduced to proving that

$$
|\xi| \leq \lim _{k \rightarrow \infty} \sigma_{f_{k}}
$$

for any point $\xi \in \operatorname{supp} \tilde{f}(\xi)$.

Assume the contrary, that (8) is not satisfied. Then there exist a point $\xi_{0} \in \operatorname{supp} \tilde{f}(\xi)$, a number $\varepsilon_{0}>0$, and a subsequence $\left\{k_{m}\right\}$ (for simplicity of notation we assume that $\left.\xi_{0}>0, k_{m}=m, m=1,2, \ldots\right)$ such that

$$
\sigma_{f_{m}} \leq \xi_{0}-2 \varepsilon_{0}, \quad m=1,2, \ldots
$$

On the other hand, it is well known that

$$
\|f(x+y)-f(x)\|_{p} \rightarrow 0, \quad|y| \rightarrow 0 .
$$

It obviously follows from (5) and (10) that

$$
\left\|f_{k}-f\right\|_{p} \rightarrow 0, \quad k \rightarrow \infty
$$

therefore, $f_{k}$ converges weakly to $f$ in $\mathscr{S}^{\prime}$, and therefore $\tilde{f}_{k}$ also converges weakly to $\tilde{f}$ in $\mathscr{S}^{\prime}$.

Now we choose a function $\varphi(x) \in C_{0}^{\infty}(\mathbf{R})$ such that $\langle\tilde{f}, \varphi\rangle \neq 0, \operatorname{supp} \varphi(x) \subset$ $\left[\xi_{0}-\varepsilon_{0}, \xi_{0}+\varepsilon_{0}\right]$. Then it follows readily from $\tilde{f}_{m} \rightarrow \tilde{f}$ weakly in $\mathscr{S}^{\prime}$ and (9) that

$$
0=\left\langle\tilde{f}_{m}, \varphi\right\rangle \rightarrow\langle\tilde{f}, \varphi\rangle \neq 0, \quad m \rightarrow \infty .
$$

We thus arrive at a contradiction. The proof is complete.

We close this paper with the following

Theorem 2. Suppose that $f(x) \in C^{\infty}(\mathbf{R})$ is an arbitrary $2 \pi$-periodic function and $1 \leq p \leq \infty$. Then there exists the limit

$$
d_{f}=\lim _{n \rightarrow \infty}\|\| f^{(n)} \|_{p}^{1 / n}
$$

and moreover

$$
d_{f}=\sigma_{f}=\sup \{|k|: k \in \operatorname{supp} \tilde{f}(\xi)\},
$$

where $\||\cdot|\|_{p}$ is the $L^{p}(0,2 \pi)$-norm.

Proof. Representing the function $f(x)$ by its Fourier series, we have

$$
f(x)=\sum_{k=-\infty}^{\infty} f_{k} \exp (i k x)
$$

where

$$
f_{k}=(2 \pi)^{-1}(f, \exp (-i k x)), \quad k=0, \pm 1, \ldots
$$

Therefore,

$$
f^{(n)}(x)=\sum_{k=-\infty}^{\infty} f_{k}(i k)^{n} \exp (i k x), \quad n=0,1, \ldots
$$


Hence, in view of the Hölder inequality,

$$
\begin{aligned}
\left|f_{k} k^{n}\right| & =(2 \pi)^{-1}\left|\left(f^{(n)}, \exp (-i k x)\right)\right| \\
& \leq(2 \pi)^{-1 / p}||\left|f^{(n)}\right| \|_{p},
\end{aligned}
$$

where $n=0,1, \ldots ; k=0, \pm 1, \ldots$.

Consequently,

$$
\lim _{n \rightarrow \infty}\left|f_{k} k^{n}\right|^{1 / n}=|k| \leq \underline{\lim }_{n \rightarrow \infty}||\left|f^{(n)}\right| \|_{p}^{1 / n}
$$

for any index $k$ such that $f_{k} \neq 0$.

Using

$$
\tilde{f}(\xi)=\sum_{k=-\infty}^{\infty} f_{k} \delta(\xi+k)
$$

and (11), we have

$$
\sigma_{f} \leq \lim _{n \rightarrow \infty}\left\|f^{(n)}\right\| \|_{p}^{1 / n} .
$$

Further, we show that

$$
\varlimsup_{n \rightarrow \infty}\left\|\left|f^{(n)}\right|\right\|_{p}^{1 / n} \leq \sigma_{f} .
$$

It is enough to prove (13) for $\sigma_{f}<\infty$. Then by the Paley-Wiener-Schwartz theorem it follows that $f$ is an analytic function of exponential type $\leq \sigma_{f}$. Hence, it follows from the inequality of Bernstein and Nikolsky that

$$
\|\| f^{(n)}\left|\left\|_{p} \leq \sigma_{f}^{n}\right\|\right| f \|_{p}, \quad n=0,1, \ldots,
$$

and (13) is an immediate consequence of the last inequalities.

Combining (12) and (13) yields

$$
\underline{\lim }_{n \rightarrow \infty}||\left|f^{(n)}\right|\left\|_{p}^{1 / n}=\varlimsup_{n \rightarrow \infty}\left|\left\|f^{(n)} \mid\right\|_{p}^{1 / n}=\sigma_{f} .\right.\right.
$$

The theorem is proved.

\section{REFERENCES}

1. A. N. Kolmogoroff, On inequalities between upper bounds of the successive derivatives of an arbitrary function on an infinite interval, Amer. Math. Soc. Transl. 4 (1949).

2. E. M. Stein, Functions of exponential type, Ann. of Math. (2) 65 (1957), 582-592.

3. S. M. Nikolsky, Approximation of functions of several variables and imbedding theorems, "Nauka", Moscow, 1977.

Institute of Mathematics, P. O. Box 631, Bo Ho, Hanoi, Vietnam 